\title{
CAR T-Cell Therapy for Large B-Cell Lymphoma
}

\author{
MARITZA C. ALENCAR, DNP, MBA, APRN-BC, BMTCN ${ }^{\circledR}$
}

From UM Sylvester Comprehensive Cancer Center, Miami, Florida

Author's disclosures of conflicts of interest are found at the end of this article.

Correspondence to: Maritza C. Alencar, DNP, MBA, APRN-BC, BMTCN ${ }^{\circledast}$, UM Sylvester Comprehensive Cancer Center, 1400 NW 10th Avenue, \#605, Miami, Florida 33136

E-mail: mcalencar@miami.edu

https://doi.org/10.6004/jadpro.2019.10.4.9

(c) 2019 Harborside $^{\mathrm{TM}}$

\begin{abstract}
Chimeric antigen receptor (CAR) T-cell therapy represents an exciting innovation in the treatment of cancer. With the approval of two revolutionary anti-CD19 CAR T-cell therapies (axicabtagene ciloleucel [Yescarta] and tisagenlecleucel [Kymriah]) for relapsed/refractory large B-cell lymphoma, health-care providers must consider how to prepare for the future of cancer care. While these therapies have been shown to provide clinical benefit to many patients, they are associated with a range of adverse events (AEs) that will need to be identified, assessed, and managed. Widespread education of health-care providers, patients, and caregivers will also be essential to ensure the success of this novel treatment. This supplement aims to provide practical information to the advanced practitioner on a number of considerations relating to the implementation and expansion of CAR T-cell therapy, including CAR T-cell biology and mechanism of action, how to identify, grade, and manage treatment-related AEs, as well as best practices for educating patients and caregivers about this type of therapy.
\end{abstract}

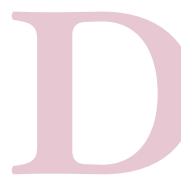

espite significant improvement in therapies for patients with relapsed/refractory large B-cell lymphoma over the past decade, treatment remains challenging and outcomes are often poor (Crump et al., 2017). More effective treatment options are clearly needed. Anti-CD19 chimeric antigen receptor (CAR) $\mathrm{T}$ cell therapy represents an exciting innovation in the treatment of these patients. This novel cellular immunotherapy reprograms the specificity and function of $T$ cells for the treatment of large B-cell lymphoma (Davila, 2017).
At the time of publication, there are currently two commercially available anti-CD19 CAR T-cell therapies. Axicabtagene ciloleucel (Yescarta) is indicated for adults with relapsed or refractory large B-cell lymphoma after more than two lines of therapy. This includes diffuse large B-cell lymphoma (DLBCL), primary mediastinal large B-cell lymphoma, DLBCL arising from follicular lymphoma, and high-grade B-cell lymphoma. It is not indicated for patients with primary central nervous system lymphoma (Kite Pharma Inc., 2017). Recently, tisagenlecleucel (Kymriah) 
was also approved for the treatment of DLBCL, DLBCL arising from follicular lymphoma, and high-grade B-cell lymphoma. Tisagenlecleucel is also indicated for the treatment of refractory Bcell precursor acute lymphoblastic leukemia in patients up to 25 years of age (Novartis Pharmaceuticals Corporation, 2018). In the first article of this supplement, Kathleen McDermott, RN, BSN, $\mathrm{OCN}^{\circledR}, \mathrm{BMTCN}^{\circledR}$, and Lauren Spendley, AGNP, MSN, AOCN ${ }^{\circledR}$, discuss these treatments, their mechanism of action, and the clinical data (McDermott, \& Spendley, 2019).

With two revolutionary cancer treatments now available, health-care providers must consider how to prepare for the future of cancer care. This supplement aims to provide guidance on a number of considerations when making these preparations (Figure 1).

\section{IDENTIFYING, ASSESSING, AND MANAGING ADVERSE EVENTS}

While CAR T-cell therapies have been shown to provide clinical benefit in many patients, they have also been associated with a range of adverse events (AEs) of varying severity and duration. Two of the most clinically significant AEs are cytokine release syndrome (CRS) and neurologic events (also known as CAR T-cell-related encephalopathy syndrome [CRES] or, most recently, immune effector cell-associated neurotoxicity syndrome [ICANS]; Lee et al., 2018; Neelapu et al., 2017).

It is important for clinical programs to develop standardized documentation tools via electronic health records to assist with capturing the signs and symptoms of these AEs. Institutions will also need to determine which grading scale will be used to determine $\mathrm{AE}$ severity. Various scales can be used to grade CRS, including the Penn grading scale (Porter, Frey, Wood, Weng, \& Grupp, 2018), the Lee grading scale (Lee et al., 2014), and the Common Terminology Criteria for Adverse Events (CTCAE; U.S. Department of Health and Human Services, 2017). Additionally, a multi-institutional group of experts convened to develop a grading scale that included CRS and neurologic event symptoms called CAR T-cell therapy-associated TOXicity (CARTOX; Neelapu et al., 2017). To standardize grading across all institutions for both clinical trials and the grading of toxicities in patients receiv- ing commercial products, the American Society for Blood and Marrow Transplantation (ASBMT) has recently published consensus grading for CRS and neurologic toxicity associated with immune effector cells (Lee et al., 2018). While each CRS scale is unique, all are geared to recognizing mild to lifethreatening signs of CRS and are intended to guide treatment. Recommendations for AE grading and management will continue to evolve as experience with the technology deepens, including further standardization and unification of CRS and neurologic event guidelines.

Health-care providers working with patients receiving CAR T-cell therapy must be astute at recognizing the signs and symptoms of AEs post therapy. Early detection of abnormal signs leads to early intervention and prevention of any delays in therapy (McGuirk et al., 2017). Signs and symptoms such as tachycardia, fever, pain, or neurologic changes need to be understood prior to initiating a CAR T-cell therapy program (McGuirk et al., 2017). Early intervention will allow the health-care team to set a plan and accurately treat patients post infusion when the onset of CRS and neurologic events usually occurs. Approximately $30 \%$ to $94 \%$ of patients will develop CRS within the first 3 weeks, and $20 \%$ to $64 \%$ will develop CRES or ICANS neurologic events that can last up to 4 weeks (Wang \& Han, 2018).

In the second article of this supplement, Sherry Adkins, RN, MSN, ANP-C, provides further details on AEs and their management, including a CRS case study (Adkins, 2019).

\section{CLINICAL PRACTICE GUIDELINES}

The development of clinical practice guidelines for practitioners can also be useful in guiding the new or seasoned advanced practitioner in managing patients receiving CAR T-cell therapy. These guidelines form a framework of best practices for clinical decision-making and improving patient outcomes (Graham \& Harrison, 2005). Given the limited and evolving information currently available on CAR T-cell therapy, it is vital for clinical programs to frequently review and revise clinical practice guidelines to reflect the latest evidence. In particular, for AE management, a standardized mechanism for assessing and documenting AEs promotes continuity of care and smooth commu- 
nication across the multidisciplinary team (Reimschissel et al., 2017).

\section{WIDESPREAD EDUCATION}

It is essential that the health-care team directly involved with patients receiving CAR T-cell therapy be well educated and prepared to mitigate situations that may be encountered throughout the CAR T-cell therapy process (McGuirk et al., 2017). For example, infusion center staff have a pivotal role in preparing patients to receive CAR T-cell therapy. In many institutions, infusion centers provide lymphodepleting chemotherapy prior to administering CAR T-cell therapy. These healthcare providers must be educated on the lymphodepleting agents that will be utilized, as well as how to recognize the signs and symptoms of significant AEs (McGuirk et al., 2017).

It is equally important for community providers to be educated on the CAR T-cell therapy process and management of patients. Clinical competence must be developed through collaborations between specialty centers offering CAR T-cell therapy. Care coordination across settings and team-based care is important to ensure that all involved parties can assist patients at any given time (Smith, 2018).

Patients are typically transitioned back to their community providers for follow-up at approximately 30 days post infusion. In some instances, patients may end up in the emergency department for a variety of reasons. Health-care providers in these settings also need to be prepared to manage symptoms associated with CRS or neurologic events (McGuirk et al., 2017). Family members and caregivers must also be vigilant in reporting AEs. Consistent widespread education needs to occur and will lead to an improved comfort level for community providers who may encounter a CAR T-cell therapy recipient in their clinic or hospital.

In the third article of this supplement, Alix Beaupierre, RN, BSN, OCN ${ }^{\circledR}$, Nicole Kahle, MS, $\mathrm{RN}, \mathrm{OCN}^{\circledR}, \mathrm{BMTCN}^{\circledR}$, Rachel Lundberg, PA-C, MPAS, and Amy Patterson, MSN, APRN, AOCNS ${ }^{\circledR}$, BMTCN $^{\circledR}$, provide an overview of the coordination of care required for CAR T-cell therapy and best practice examples of education for multidisciplinary care teams, patients, and caregivers (Beaupierre, Kahle, Lundberg, \& Patterson, 2019).

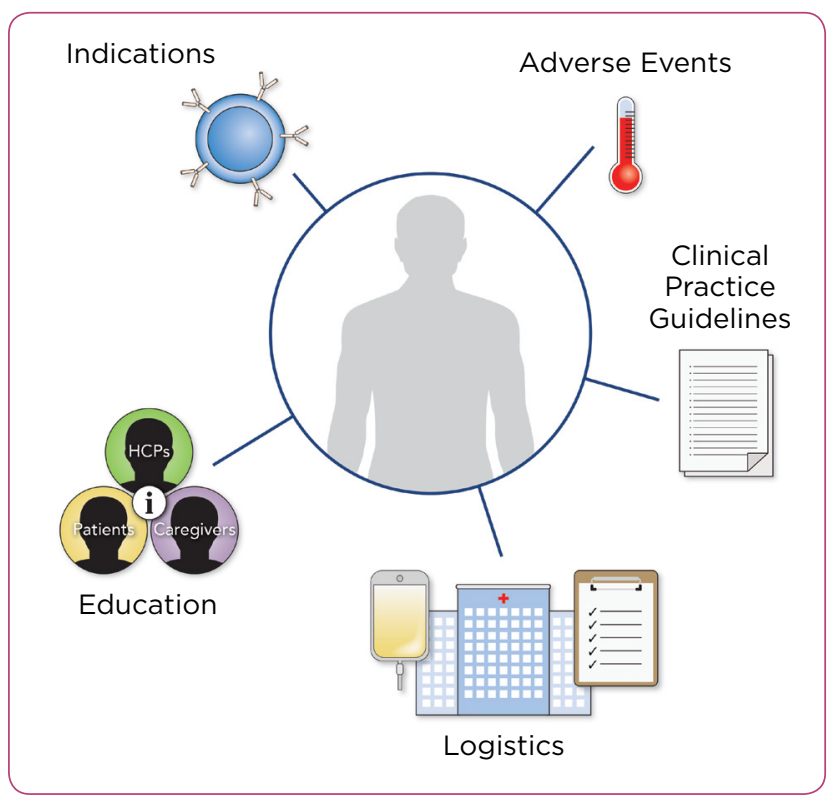

Figure 1. Preparing for the future: Considerations when implementing a CAR T-cell therapy program. HCP = health-care provider.

\section{ADVANCED EDUCATION OPPORTUNITIES}

The ever-changing field of hematology and oncology creates opportunities for specialty training programs for advanced practice providers and nurses alike. Fellowship and residency programs can augment the knowledge base of nurses beyond the school setting. Specifically, in the advanced practice nurse setting, graduate-level education is focused mainly on primary care. One such program is the Oncology Nurse Practitioner Fellowship at the University of Miami Sylvester Comprehensive Cancer Center (Alencar, Butler, MacIntyre, \& Wempe, 2018). They offer a blood and marrow transplant with cellular therapy training that spans 50 weeks. This allows the nurse practitioner to be immersed in the complexities surrounding this patient population and hone his or her skills, learning from a team of experts who have been involved with the CAR T-cell therapy population since the ZUMA-1 pivotal trial.

These types of programs also ensure that new nurses or advanced practitioners are prepared for the full scope of their roles (Nevidjon et al., 2010). The program could potentially promote increased job satisfaction, decreased turnover, high proficiency, decreased burnout, and highquality cancer care. 


\section{OVERCOMING LOGISTICS OF CAR T-CELL THERAPY}

While the initial approvals of axicabtagene ciloleucel and tisagenlecleucel in 2017 were groundbreaking, they also highlighted the many logistical obstacles to developing a CAR T-cell therapy program. Institutions with current CAR T-cell therapy programs have described their journeys. A common initial step undertaken by the first programs was to identify each component of the process and create a workflow. Memorial Sloan Kettering Cancer Center has successfully implemented and published their workflow (Brown \& Mahmoudjafari, 2018).

Creating workflows that identify resources available in the institution is imperative in planning how to strategically roll out a CAR T-cell therapy program. Appropriate planning that involves key stakeholders lays the path for a successful program. As CAR T-cell therapy matures and team members become more experienced, programs will need to refine workflows to reflect the most current practice data. Comprehension of the entire CAR T-cell therapy workflow process and how each member of the health-care team affects each step can lead to improved patient management.

\section{OVERCOMING DIFFERENCES BETWEEN FACILITY RESOURCES}

An important caveat is differentiating resources that are available from center to center. Depending on available resources, a center may need to develop creative and unique ways for implementation and ensure that all key safety measures are in place throughout program workflows, while also considering the future. Programs must engage in continuous quality improvement efforts as new data arise and dictate changes that must be reflected in clinical practices and data reporting.

\section{CONCLUSION}

As the clinical use of CAR T-cell therapy increases, advanced practitioners will be highly involved in preparing institutions to implement and expand this exciting technology. They will need to understand the underlying biology of CAR T-cell therapy and will have a crucial role in identifying individuals at risk of treatment-related AEs, as well as grading and managing these AEs. Advanced practitioners will also be essential in educating patients and caregivers about this type of therapy. We hope that this supplement provides practical guidance to advanced practitioners in preparing for the future of cancer treatment.

\section{Acknowledgment}

Medical writing support was provided by Katherine R. Nibouar, PhD, of Nexus Global Group Science LLC, sponsored by Kite, a Gilead Company.

\section{Disclosure}

This manuscript was sponsored by Kite, a Gilead Company. Ms. Alencar serves on the speakers bureau for Kite, a Gilead Company.

\section{References}

Adkins, S. (2019). CAR T-cell therapy: Adverse events and management. Journal of the Advanced Practitioner in Oncology, 10(suppl 3), 21-28. https://doi.org/10.6004/ jadpro.2018.10.4.11

Alencar, M. C., Butler, E., MacIntyre, J., \& Wempe, E. P. (2018). Nurse practitioner fellowship: Developing a program to address gaps in practice. Clinical Journal of Oncology Nursing, 22(2), 142-145. https://doi.org/10.1188/18. CJON.142-145

Beaupierre, A., Kahle, N., Lundberg, R., \& Patterson, A. (2019). Educating multidisciplinary care teams, patients, and caregivers on CAR T-cell therapy. Journal of the Advanced Practitioner in Oncology, 10(suppl 3), 29-40. https://doi.org/10.6004/jadpro.2018.10.4.12

Brown, M. A., \& Mahmoudjafari, Z. (2018). CAR-T cell program development: A tale of two institutions. Hematology/Oncology Pharmacy Association (HOPA) News, 15(3), 7. Retrieved from http://www.hoparx.org/hopa-news/ volume-15-issue-3-2018/feature-car-t-cell-program-development-a-tale-of-two-institutions

Crump, M., Neelapu, S. S., Farooq, U., Van Den Neste, E., Kuruvilla, J., Westin, J.,...Gisselbrecht, C. (2017). Outcomes in refractory diffuse large B-cell lymphoma: Results from the international SCHOLAR-1 study. Blood,130(16), 18001808. https://doi.org/10.1182/blood-2017-03-769620

Davila, M. L. (2017). The latest advances in CAR T-cell therapy for refractory and relapsed lymphomas and leukemias. Journal of the Advanced Practitioner in Oncology, 8(suppl 2), 3-13. https://doi.org/10.6004/jadpro.2017.8.6.10

Graham, I. D., \& Harrison, M. B. (2005). Evaluation and adaptation of clinical practice guidelines. Evidence-Based Nursing, 8(3), 68-72. Retrieved from http://dx.doi. org/10.1136/ebn.8.3.68

Kite Pharma Inc. (2017). Yescarta (axicabtagene ciloleucel) package insert. Retrieved from https://www.yescarta. com/files/yescarta-pi.pdf

Lee, D. W., Gardner, R., Porter, D. L., Louis, C. U., Ahmed, N., Jensen, M.,...Mackall, C. L. (2014). Current concepts in the diagnosis and management of cytokine release syndrome. Blood, 124(2), 188-195. https://doi.org/10.1182/ 
blood-2014-05-552729

Lee, D. W., Santomasso, B. D., Locke, F. L., Ghobadi, A., Turtle, C. J., Brudno, J. N.,...Neelapu, S. S. (2018). ASBMT consensus grading for cytokine release syndrome and neurological toxicity associated with immune effector cells. Biology of Blood and Marrow Transplantation, 25(4), 625638. https://doi.org/10.1016/j.bbmt.2018.12.758

McDermott, K., \& Spendley, L. (2019). Anti-CD19 CAR T-cell therapy for adult patients with refractory large B-cell lymphoma. Journal of the Advanced Practitioner in Oncology, 10(suppl 3), 11-20. https://doi.org/10.6004/jadpro.2018.10.4.10

McGuirk, J., Waller, E., Qayed, M., Abhyankar, S., Ericson, S., Holman, P.,...Myers, G. D. (2017). Building blocks for institutional preparation of CTL019 delivery. Cytotherapy, 19(9), 1015-1024. https://doi.org/10.1016/j. jcyt.2017.06.001

Neelapu, S. S., Tummala, S., Kebriaei, P., Wierda, W. G., Gutierrez, C., Locke, F. L.,...Shpall, E. (2017). Chimeric antigen receptor T-cell therapy - assessment and management of toxicities. Nature Reviews Clinical Oncology, 15(1), 47-62. https://doi.org/10.1038/nrclinonc.2017.148

Nevidjon, B., Rieger, P., Miller Murphy, C., Rosenzweig, M. Q., McCorkle, M. R., \& Baileys, K. (2010). Filling the gap: Development of the oncology nurse practitioner workforce. Journal of Oncology Practice, 6(1), 2-6. https://doi. org/10.1200/JOP.091072
Novartis Pharmaceuticals Corporation. (2018). Kymriah (tisagenlecleucel) package insert. Retrieved from https:// www.pharma.us.novartis.com/sites/www.pharma. us.novartis.com/files/kymriah.pdf

Porter, D., Frey, N., Wood, P. A., Weng, Y., \& Grupp, S. A. (2018). Grading of cytokine release syndrome associated with the CAR T cell therapy tisagenlecleucel. Journal of Hematology \& Oncology, 11(1), 35. https://doi.org/10.1186/ s13045-018-0571-y

Reimschissel, E., Cruz, B. D., Gonzalez, M., Buitrago, J., Goodman, C., \& Johnston, P. A. (2017). Immunotherapy toxicities: A new electronic documentation template to improve patient care. Clinical Journal of Oncology Nursing, 21(2 supplement), 41-44. https://doi.org/10.1188/17. CJON.S2.41-44

Smith, S. (2018). How to get your specialty practice ready for CAR-T cell therapies. Retrieved from https://www. mckesson.com/blog/how-to-get-your-specialty-practice-ready-for-car-t-cell-therapies/

U.S. Department of Health and Human Services. (2017). Common Terminology Criteria for Adverse Events (CTCAE) Version 5.0. Retrieved from https://ctep.cancer.gov/protocoldevelopment/electronic_applications/ctc.htm

Wang, Z., \& Han, W. (2018). Biomarkers of cytokine release syndrome and neurotoxicity related to CAR-T cell therapy. Biomarker Research, 6, 4. https://doi.org/10.1186/ s40364-018-0116-0 